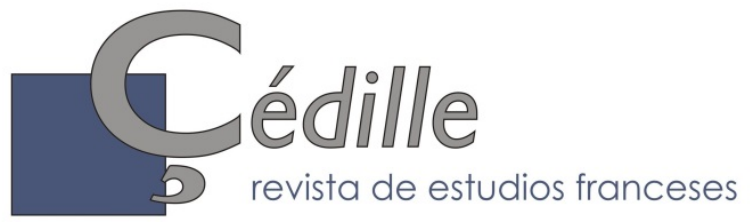

ISSN: $1699-4949$

no 17 (otońo de 2020)

Monografías 10

Les euphémismes dans les médias : entre voilements, démasquages et discours qui les traversent

Montserrat López Díaz \& Annabelle Seoane, éditrices scientifiques

\title{
Polyphonie divergente et mise en cause des euphémismes dans la presse écrite
}

\author{
Marc BONHOMME \\ Universität Bern \\ marc.bonhomme@rom.unibe.ch \\ ORCID : 0000-0003-0494-5872
}

\section{Resumen}

Adoptando un enfoque pragmático, este estudio se centra en un caso particular de asunción del eufemismo en la prensa francófona escrita: aquel en el cual, en lugar de adherirse a sus efectos atenuantes, el discurso periodístico manifiesta un posicionamiento crítico contrario. Este es tratado en términos de "polifonía divergente", definida como una confrontación de puntos de vista en desacuerdo. Después de haber situado la polifonía divergente en relación con el funcionamiento general del dialogismo y de la polifonía, este artículo pone de relieve cómo se ubica en el núcleo del cuestionamiento de los eufemismos en periódicos franceses y suizos francófonos recientes. Por una parte, analizamos sus principales modalidades discursivas, ya sea las que se relacionan con su anclaje dialógico, su textualización o sus marcas enunciativas. Por otra, examinamos las diferentes motivaciones (comunicativas, referenciales o socio-discursivas) del rechazo polifónico de los eufemismos en los periódicos seleccionados. Además, destacamos las estrategias argumentativas en las que se inscribe la polifonía divergente con sus procedimientos para desenmascarar y estigmatizar los eufemismos perseguidos.

Palabras clave: Dialogismo. Eufemismo. Punto de vista, Polifonía divergente. Posicionamiento crítico. Estrategia argumentativa.

\section{Résumé}

Adoptant une approche pragmatique, la présente étude porte sur un cas particulier de prise en charge des euphémismes dans la presse francophone écrite : celui où, au lieu d'adhérer à leurs effets d'atténuation, le discours journalistique manifeste un positionnement critique à leur encontre. Ce positionnement est envisagé en terme de "polyphonie divergente ", définie comme une confrontation de points de vue en désaccord. Après avoir situé la polyphonie divergente par rapport au fonctionnement général du dialogisme et de la polyphonie, cet article montre comment elle est au cœur de la mise en cause des euphémismes dans des journaux français et suisses romands récents. D'une part, nous analysons les principales modalités discursives de cette polyphonie divergente, qu'elles concernent son ancrage

* Artículo recibido el 17/10/2019, aceptado el 16/04/2020. 
dialogique, sa mise en texte ou ses marquages énonciatifs. D'autre part, nous examinons les différentes motivations (communicationnelles, référentielles ou sociodiscursives) du rejet polyphonique des euphémismes dans les journaux retenus. Par ailleurs, nous mettons en évidence les stratégies argumentatives dans lesquelles s'inscrit la polyphonie divergente avec ses procédures de démasquage et de stigmatisation des euphémismes pris pour cible.

Mots-clefs : Dialogisme. Euphémisme. Point de vue. Polyphonie divergente. Positionnement critique. Stratégie argumentative.

\begin{abstract}
Adopting a pragmatic approach, this study focuses on a particular case of taking charge of euphemisms in the French-speaking print press : the one where, instead of adhering to their mitigation effects, the journalistic discourse displays a critical position against them. This one is envisaged in terms of "divergent polyphony", defined as a confrontation of points of view in dissension. After having located the divergent polyphony in relation to the general functioning of dialogism and polyphony, this paper shows how it is at the heart of the questioning of euphemisms in recent French and Swiss French-speaking newspapers. On the one hand, we analyze its main discursive modalities, whether they concern its dialogical anchorage, its setting in text or its enunciative marks. On the other hand, we examine the different motivations (communicational, referential or sociodiscursive) for the polyphonic rejection of euphemisms in the selected newspapers. Furthermore, we highlight the argumentative strategies in which the divergent polyphony fits with its unmasking and stigmatizing procedures against the euphemisms targeted.
\end{abstract}

Keywords : Dialogism. Euphemism. Point of view. Divergent polyphony. Critical positioning. Argumentative strategy.

\title{
0. Introduction
}

Habituellement défini comme "un procédé d'atténuation consistant à éviter d'énoncer des idées désagréables ou déplaisantes" (Pougeoise, 2001: 127), l'euphémisme est une figure rhétorique en vogue, à en juger par les nombreuses publications qui lui ont été dernièrement consacrées ${ }^{1}$. Par ailleurs, il donne lieu à des approches disparates, qu'elles soient sociolinguistiques (Courthéoux, 2005), sémantiques (Casas Gómez, 2005) ou stylistiques (Jaubert (2008). Cependant, depuis quelques années l'étude de l'euphémisme bénéficie d'un renouveau théorique avec le courant pragmatique, attentif à ses conditions d'utilisation en contexte et à ses effets d'estompage suivant les situations de discours ${ }^{2}$.

\footnotetext{
${ }^{1}$ Voir entre autres Druetta \& Paissa (2009), Jamet \& Jobert (2010) ou López Díaz \& Sablayrolles (2016).

${ }^{2}$ Voir à ce propos Bonhomme, de la Torre \& Horak (2012).
} 
Adoptant cette approche méthodologique de la pragmatique, nous nous proposons d'approfondir le fonctionnement de l'euphémisme dans l'un des genres discursifs qui l'exploite le plus : la presse écrite ${ }^{3}$. Dans la majorité des cas, les euphémismes de presse fonctionnent sous un régime consensuel selon lequel leur production ou leur retraitement par les acteurs médiatiques s'accompagnent d'une adhésion à leurs configurations dédramatisantes de la réalité. Mais il arrive également que l'usage des euphémismes dans la presse alimente des positionnements critiques qui se distancient de leurs effets d'atténuation. Ce sont ces positionnements critiques qui feront l'objet de cette étude, à travers leur examen dans un cadre polyphonique sur la base d'un corpus récent tiré de la presse écrite française et suisse ${ }^{4}$. Après une présentation de ce cadre polyphonique dans lequel s'inscrivent un certain nombre d'euphémismes de presse, nous verrons en quoi leur mise en cause constitue un phénomène de polyphonie divergente. Puis nous analyserons les modalités discursives, ainsi que les motivations et les stratégies argumentatives d'une telle polyphonie divergente.

\section{L'euphémisme comme constellation énonciative}

Figure référentielle estompant certaines réalités problématiques, l'euphémisme a longtemps été envisagé comme un procédé discursif individualisé, émanant de la performance stylistique d'un locuteur, écrivain ou autre. Or il constitue fondamentalement une constellation énonciative condensant en son sein des formes plurielles de discours. Il est avant tout une figure sociodiscursive du fait qu'il naît généralement dans l'énonciation d'un groupe ou d'une collectivité. On euphémise en effet sous la pression d'autrui et à l'intention d'autrui, la voix de l'Autre étant omniprésente lors de la production d'un euphémisme ${ }^{5}$. Plus largement - et notamment avec la presse écrite caractérisée par le flux continu de l'information, l'euphémisme est une figure interdiscursive, en ce qu'il est indissociable de la circulation des discours et de leurs

\footnotetext{
3 Travail réalisé dans le cadre des projets de recherche FFI2013-42249P et FFI2017-85141P (FEDER, Ministerio de Ciencia, Innovación y Universidades, AEI).

${ }^{4}$ Ce corpus est composé de cinq journaux et magazines français (Le Canard enchaîné, Le Figaro, Paris Match, Le Point, Le Progrès) et de sept journaux suisses romands (La Liberté, Le Matin, Le Matin Dimanche, Le Quotidien jurassien, Le Temps, 20 minutes et 24 heures) parus entre 2000 et 2017.

${ }^{5}$ La nature sociodiscursive de l'euphémisme explique qu'il soit très difficile d'en identifier la source première. Si l'on prend la formulation euphémique "dommages collatéraux " (pour désigner les ratés des bombardements aériens) apparue dans les années 1990 avec la guerre du Golfe, on peut sans doute cibler quelques-uns de ses énonciateurs, mais il est pratiquement impossible de savoir qui a créé ce néologisme diffusé par les autorités militaires et la presse.
} 
reprises dans des occurrences spécifiques ${ }^{6}$. Tout ceci lui confère une dimension éminemment dialogique, dans l'acception interactionnelle que donne Vion (1992) à ce terme, à savoir une ouverture sur différents discours. Cette ouverture peut s'exercer sur du dire antérieur, auquel cas on a affaire à du dialogisme "interdiscursif » (Détrie et al., 2001 : 84). Mais l'euphémisme est aussi ouvert sur des interactions postérieures toujours possibles, selon le processus du dialogisme " interlocutif " pareillement décrit par Détrie et al. (ibid.). Si le dialogisme interdiscursif inhérent à l'euphémisme a été mis en évidence, en particulier par Jaubert (2008), à travers ses recyclages de formulations édulcorées produites dans l'énonciation partagée d'une communauté, son dialogisme interlocutif pâtit encore d'un manque de considération, en dépit de quelques études sur la réception euphémique (voir Krieg-Planque, 2004 ou Bonhomme, 2012).

Tout en ayant un fonctionnement dialogique global, l'euphémisme possède une forte dimension polyphonique davantage localisée car elle opère au sein de son noyau figural. En effet, si l'on se situe dans le cadre théorique élaboré par Ducrot (1984) et Rabatel (2006), autant le dialogisme constitue un phénomène interactionnel, autant la polyphonie se présente comme un phénomène énonciatif, définissant la coprésence de différents points de vue ${ }^{7}$ ou de plusieurs voix à l'intérieur d'un même segment de discours ${ }^{8}$. Cette imbrication de points de vue énonciatifs s'effectue selon deux démarches.

D'un côté, il relève de la polyphonie convergente ${ }^{9}$ lorsqu'un événement discursif, en l'occurrence un euphémisme, implique simultanément plusieurs points de vue énonciatifs, certes hétérogènes, mais qui suivent une même orientation argumentative, comme dans cet énoncé :

[1] Les CFF prennent mieux en compte la situation des personnes à mobilité réduite (24 heures du 08/07/2015).

\footnotetext{
${ }^{6}$ Sous cet angle, l'euphémisme apparaît comme un cas particulier de discours rapporté. Sa dimension interdiscursive a été soulignée par Krieg-Planque (2003) lors de son enquête sur l'euphémisme "purification ethnique » et ses variantes dans les médias yougoslaves. De plus, par son interdiscursivité et par sa relative stabilité à travers ses occurrences, l'euphémisme se rapproche de la notion de "formule ", telle qu'elle a été théorisée par Krieg-Planque (2009).

${ }^{7}$ La polyphonie combine ainsi les critères de la pluralité et de l'altérité énonciative.

${ }^{8}$ Rappelons que les relations entre dialogisme et polyphonie sont confuses au regard de plusieurs linguistes. En effet, les uns comme Perrin (2004) privilégient la polyphonie au détriment du dialogisme ; les autres au contraire, tel Bres (2005), promeuvent celui-ci. Certains enfin, à l'exemple de Courlescu (2014), ne différencient pas conceptuellement les deux termes. Notre hiérarchisation de ces derniers d'après le double critère global/local et interactionnel/énonciatif rejoint la position adoptée par Amossy (2005).

${ }^{9}$ On trouve une première formulation de ce concept dont nous sommes l'instigateur dans Bonhomme (2005).
} 
La formulation "personnes à mobilité réduite " est identifiée comme un euphémisme dans nos représentations communes, en ce qu'elle vise à édulcorer la désignation des personnes présentant un handicap physique. Dans cette occurrence, elle met en jeu deux points de vue : le point de vue citant du journaliste et le point de vue cité de la doxa actuelle en matière de handicap. Or ces points de vue sont en accord ou co-orientés, le je-dis du journaliste assumant le on-dit de la doxa ${ }^{10}$, tout en restant en retrait par rapport à lui. S'intégrant dans le champ du dialogisme interdiscursif, cette expression n'est qu'une reprise, à l'intérieur d'un nouveau contexte, de l'interdiscours médiatico-médical prédominant. La responsabilité d'une telle expression est diluée dans une prise en charge plurielle, composée de l'ensemble des énonciateurs (médecins, responsables politiques, journalistes, etc.) qui cherchent à s'adapter au sentiment général prévalant dans le domaine de la santé depuis trois ou quatre décennies. De surcroît, la prise en charge de cet euphémisme par une énonciation polyphonique convergente favorise sa réussite auprès de ses destinataires, vu que ses effets modérateurs sont acceptés par le plus grand nombre, dans un consensus conformiste qui préserve l'équilibre rituel de la communication médiatique.

Mais un événement discursif comme l'euphémisme peut également susciter une polyphonie divergente lorsque l'énoncé met en scène plusieurs points de vue énonciatifs qui sont en désaccord ou argumentativement anti-orientés. Soit l'exemple ci-après du Progrès de Lyon :

[2] Les bourses chinoises ont fait les frais d'un nouveau mécanisme mis en place après le krach de l'été. Ce dispositif prévoit que lorsque l'indice CS1300 perd ou gagne 7\%, les échanges sont suspendus pour le reste de la séance afin d'éviter des «risques systémiques », c'est-àdire un mouvement de panique (Le Progrès du 05/01/2015).

Dans cet extrait, le journaliste reprend l'euphémisme "risques systémiques " coutumier des milieux financiers, en le reformulant par le marqueur explicatif " c'està-dire ». Mais c'est pour s'en distancier avec un positionnement critique. Au point de vue dédramatisant associé à l'expression "risques systémiques » il oppose un point de vue dramatisant, identifiable dans sa reformulation en "mouvement de panique ${ }^{11}$. Une telle polyphonie divergente, par laquelle le journaliste met en cause le bien-fondé d'un euphémisme, désamorce inévitablement ses effets illocutoires d'atténuation, tout en témoignant d'un détachement énonciatif qui en exhibe le caractère inapproprié.

\footnotetext{
${ }^{10}$ L'absence de guillemets constitue un indice de la prise en charge de cet euphémisme, mais elle n'en est pas un marqueur inhérent, dans la mesure où un euphémisme non assumé peut lui aussi être dépourvu de signe typographique (voir les exemples [17] ou [25]).

${ }^{11}$ Dans ce cas, la divergence polyphonique provient d'un désaccord concernant l'échelle de l'intensité ("systémique " s'inscrit sur un seuil plus bas que " panique ») et secondairement l'échelle des affects, la convocation des « risques » étant psychiquement moins traumatisante que celle de la panique.
} 
Ce sont ces procédures de désamorçage qui retiendront désormais notre attention, à travers leurs principales réalisations.

\section{Modalités discursives de la polyphonie divergente dans les contextes euphé- miques}

Loin de répondre à des mécanismes simples, la polyphonie divergente contestant les euphémismes de presse s'exerce sur plusieurs plans du discours, selon des modalités diversifiées et cumulatives. Quatre d'entre elles sont prédominantes.

Sur le plan dialogique régissant la dynamique des interactions verbales, la polyphonie divergente prend place dans au moins trois relations énonciatives. D'une part, elle se manifeste entre le discours du journaliste et l'interdiscours d'une source énonciative antérieure ou de la doxa, à l'image de cette occurrence :

[3] Près de 40\% des Sud-Soudanais risquent l'« insécurité alimentaire "

(20 minutes du 14/07/2015).

À travers son marquage graphique, le journaliste du quotidien 20 minutes prend ses distances avec l'euphémisme médiatico-administratif conventionnel « insécurité alimentaire " qui voile la désignation de la famine en Afrique. D’autre part, il arrive que la polyphonie divergente à l'encontre d'un euphémisme émane d'un acteur de l'actualité dont le discours du journaliste relate les propos, à l'instar de cet énoncé :

[4] Le guide édité par la ville d'Amsterdam laisse Thérèse Moreau très perplexe : "Je suis choquée d'apprendre qu'on incite les fonctionnaires à parler de "personnes roses" pour désigner les homosexuels... Les auteurs de ce guide ont-ils conscience que cette couleur était celle qu'avaient choisie les nazis, quand ils marquaient d'un triangle rose les homosexuels envoyés dans les camps?" (Le Matin Dimanche du 24/09/2017).

Dans ce passage du Matin Dimanche, hebdomadaire de Lausanne, le journaliste Michel Audétat se contente de rapporter le point de vue critique ( je suis choquée ») de la féministe suisse Thérèse Moreau sur l'euphémisme "personnes roses " que le récent guide linguistique diffusé par la ville d'Amsterdam recommande pour dénommer les homosexuels. Dans les deux cas précédents, l'énonciation polyphonique divergente se greffe sur du dialogisme interdiscursif, en ce qu'elle ne prend pas en charge du discours antérieur à l'occurrence journalistique. Mais elle se développe aussi dans le champ du dialogisme interlocutif ${ }^{12}$, dès lors que le discours du journaliste anticipe l'hostilité des lecteurs à des euphémismes médiatiques. Ainsi en est-il

${ }^{12}$ Conformément à la conception de Moirand (2002 : 176) suivant laquelle le dialogisme interlocutif définit les relations " que tout énoncé entretient avec les énoncés de compréhension-réponse des destinataires réels ou virtuels, que l'on anticipe». 
dans l'extrait suivant du Temps au sujet de l'euphémisme "dommages collatéraux " pour dénommer les victimes civiles d'une guerre :

[5] Tout lecteur sensé trouvera sans doute monstrueuse l'expression "dommages collatéraux " pour désigner les victimes des raids en Syrie, dans la mesure où elle dénie tout droit à ces victimes (Le Temps du 14/07/2016).

Par ailleurs, sur le plan de sa textualisation, la polyphonie divergente à l'encontre des euphémismes occupe des places privilégiées dans le discours de presse. Elle apparaît naturellement dans le corps des articles factuels rendant compte de l'actualité, comme on le constate avec les exemples [2] ou [3]. Mais elle est particulièrement attestée dans les sous-genres journalistiques relevant du commentaire, avec la subjectivité et la mise en débat qui les caractérisent ${ }^{13}$. En plus du courrier des lecteurs (voir les exemples [15] et [19]), les euphémismes créent de nombreux conflits polyphoniques dans le sous-genre de la titraille :

[6] Lavé de tout soupçon, le chômage s'appellera désormais «sousemploi » (Le Temps du 16/10/2000).

À travers le déploiement de ce titre du Temps, le point de vue asserté de nontravail lié au terme "chômage " cède la place au point de vue euphémique, à la fois détensif et mélioratif, de moins de travail associé au lexème "sous-emploi ", lui-même étant contesté par sa mise entre guillemets ${ }^{14}$. Le sous-genre de l'éditorial abonde pareillement en euphémismes insérés dans une contextualisation conflictuelle :

[7] Tamedia maîtrise comme personne la novlangue financière : "valorisation du contenu et consolidation du positionnement régional ». Derrière cette manipulation mentale et ce paravent d'euphémismes, l'éditeur zurichois a annoncé hier une cure drastique au sein de la rédaction de 24 heures. Vingt-deux licenciements et une atteinte sévère aux contenus (éditorial de Serge Gumy, 24 heures du 28/09/2016).

Au point de vue positif de la formulation euphémique "valorisation du contenu et consolidation du positionnement régional " s'oppose le point de vue négatif de sa réinterprétation journalistique en "cure drastique " et en "licenciements ». Le sous-genre de la chronique comporte également beaucoup de clivages polyphoniques analogues, à l'instar de celle de Martina Chyba dans Le Matin, dont le positionnement humoristique démonte l'euphémisation outrancière de la novlangue actuellement en vigueur :

${ }^{13}$ Constituant l'idéal de l'écriture journalistique (voir Agnès, 2002), la distinction entre faits et commentaires doit être envisagée en terme de dominantes et non comme une opposition tranchée.

${ }^{14}$ De plus, exprimant l'aspect non-accompli, la forme réfléchie utilisée ("s'appellera ") confère à la formulation euphémique incriminée davantage de saillance que ne le ferait la forme passive ("sera appelé »). 
[8] Impolitiquement correct

Amatrice inconditionnelle de la novlangue pédante et politiquement correcte, je me dois de partager les dernières découvertes. Déjà cet été, j'ai adoré les campings qui ne veulent plus qu'on les appelle les campings, mais qui demandent que l'on dise désormais « hôtellerie en plein air " ${ }^{15}$. Haha. J'ai aussi appris que je n'étais pas petite, mais " de taille modeste " et qu'un nain était une " personne à verticalité contrariée " Si, si. (Le Matin du 17/08/2017).

En outre, sur le plan de son actualisation discursive, la polyphonie divergente touchant les euphémismes de presse oscille entre des réalisations explicites et d'autres beaucoup plus diffuses. Tantôt en effet les points de vue antagonistes sont clairement formulés dans l'énoncé journalistique, comme dans cet exemple de La Liberté, quotidien de Fribourg :

[9] Le pape Benoît XVI demande pardon pour les "erreurs" de l'Église commises dans le passé. Mais le terme dépénalisant «erreurs » dissimule mal les exactions exercées par les catholiques contre les protestants après la Réforme ou la participation des croyants au commerce des esclaves (La Liberté du 14/08/2010).

Cette occurrence met bien en évidence les deux points de vue conflictuels en jeu autour de l'euphémisme "erreurs ", à savoir son évaluation papale non-criminelle impliquée par une telle dénomination et son évaluation criminelle qui découle de sa recatégorisation journalistique en " exactions ". Tantôt les points de vue antagonistes révélés par l'emploi d'euphémismes sont plus ou moins implicites et ne peuvent être restitués qu'à l'issue de calculs interprétatifs basés sur leur contexte et les compétences des lecteurs. C'est le cas pour cet énoncé euphémique :

[10] La Chine n'a toujours pas renoncé au fond d'elle-même à la « libération " de Taïwan (Le Temps du 07/02/2014).

Dans cet exemple, le terme "libération " suscite visiblement les réserves du journaliste, mais son statut sémantique et ses enjeux idéologiques n’apparaissent pas à la surface de l'article. À partir de notre savoir sur l'histoire chinoise, nous avons cependant la capacité de mettre à jour le conflit polyphonique que ce terme engendre. Dans la phraséologie édulcorante des autorités communistes chinoises, "libération » signifie la reconquête, politique ou militaire, de Taïwan. Mais on peut présumer que ce terme est considéré comme un euphémisme suspect aux yeux du journaliste pour lequel la main-mise de la Chine sur l'île de Taiwan est nécessairement contraignante, dans la mesure où ses habitants ne la souhaitent pas. De la sorte, le point de vue chi-

${ }^{15}$ Cet euphémisme joue essentiellement sur l'axe mélioratif, « hôtellerie » supposant un standing plus élevé que "camping". Par ailleurs, son insertion dans un cotexte directif ("qui demandent que l'on dise ») est symptomatique des contraintes sociales qui président à l'instauration d'un euphémisme. 
nois de non-contrainte apparente et le point de vue journalistique de contrainte insinuée sont non seulement donnés à entendre par cet énoncé, mais la restitution de ces positionnements énonciatifs antithétiques ne pose pas de véritables difficultés, en ce qu'ils sont déjà connus du public ${ }^{16}$, pour peu qu'il s'intéresse à la politique internationale.

De surcroît, la polyphonie divergente se matérialise dans le discours journalistique par différents marqueurs énonciatifs qui peuvent se combiner. Ressortissant à l' "hétérogénéité montrée » théorisée par Authier-Revuz (1982: 91), ceux-ci constituent des indices essentiels pour une interprétation polyphonique clivante ${ }^{17}$, voire conflictuelle, des euphémismes qu'ils affectent. Dans l'ensemble, ils se répartissent en trois catégories. Ils sont d'abord typographiques quand des guillemets, comme dans les exemples [9] et [10] vus précédemment, isolent un euphémisme sur la page. De tels guillemets sont foncièrement ambigus, opérant en même temps comme des signaux du discours euphémique rapporté, présenté sous la forme fragmentaire d'un " îlot citationnel ${ }^{18}$ " (Authier-Revuz, 1996 : 97), et comme des indices de la non-prise en charge des euphémismes mentionnés par les journalistes. De fait, ces guillemets font ressortir leur prudence, ce qui est de nature à éveiller la méfiance du public. La mise en saillance d'euphémismes se concrétise aussi par des italiques qui marquent alors un soulignement graphique attirant l'attention des lecteurs sur une formulation euphémique estimée douteuse et les invitant à la réinterpréter dans sa véritable acception avec l'aide du cotexte :

[11] La paisible Newry reste traumatisée par trois décennies de troubles, selon la terminologie officielle ${ }^{19}$, et les 3500 morts dont ils sont responsables ${ }^{20}$ (Le Point du 22/06/2017).

Les marqueurs de décrochage énonciatif affectant les euphémismes sont encore lexicaux, quand un adjectif, comme " monstrueuse » en [5], ou un nom, comme " manipulation" en [7], introduisent des jugements qui disqualifient des formulations telles que "dommages collatéraux » ou "valorisation du contenu ». Le contraste axiologique instauré entre la négativité des jugements exprimés et le point de vue

\footnotetext{
${ }^{16}$ Ici et dans les pages qui suivent, le terme public est pris dans un sens générique qui n'exclut pas que le lectorat de la presse écrite soit diversifié.

${ }^{17}$ Une interprétation polyphonique est clivante lorsqu'elle établit une simple prise de distance avec une tournure euphémique jugée tendancieuse.

${ }^{18}$ Comme le montre Bonhomme (sous presse), si l'îlot citationnel déborde la figure de l'euphémisme, il présente l'avantage de conforter son statut de formule et ainsi d'exhiber sa nature conventionnelle.

${ }^{19}$ Référant au discours institutionnel, cette formulation constitue un procédé typique de distanciation, grâce auquel les médias évitent de prendre position.

${ }^{20}$ Dans cet énoncé, la mention de nombreuses victimes et de la ville irlandaise de Newry nous incite à voir derrière les « troubles » en question la guerre civile qui a longtemps frappé l'Irlande du Nord.
} 
lénifiant attaché aux euphémismes sur lesquels ils portent déstabilise inévitablement ces derniers.

Les marqueurs de distanciation polyphonique prennent enfin la forme de locutions énonciatives plus étendues, à l'exemple de «comme on dit » qui reporte sur la doxa la responsabilité de l'euphémisme concerné, indiquant sa non-prise en charge par le discours journalistique :

[12] La guerre pour le Kosovo entre dans son troisième mois de bombardements et de destructions. Le bilan de soixante jours de " sorties ", comme on dit à Bruxelles, est objectivement désastreux, et les généraux ne parviennent plus à dissimuler leurs craintes (Le Temps du 25/05/2000).

\section{Motivations du rejet polyphonique des euphémismes}

Il importe à présent d'analyser plus précisément les relations énonciatives entre la polyphonie divergente et la critique des euphémismes dans le discours de presse écrite. Ces relations mettent en jeu différentes motivations qui expliquent leur rejet et qui mobilisent plusieurs points de vue dépréciatifs de la part d'énonciateursévaluateurs ${ }^{21}$, qu'ils soient journalistes ou acteurs de l'actualité.

Ainsi, perçu suivant un point de vue communicationnel, l'euphémisme est parfois refusé, en ce qu'il paraît constituer une entrave à la clarté et à la bonne transmission du discours. C'est le cas quand un terme euphémique anglo-saxon comme " outplacement " pour désigner un licenciement est jugé " obscur pour le grand public » dans un article du Temps :

[13] La crise économique a fortement développé l'outplacement en Suisse. Terme obscur pour le grand public et à éviter, l'outplacement est un service de reclassement offert par une entreprise qui désire se séparer d'un collaborateur (Le Temps du 11/06/2009).

Il en est de même lorsqu'une formulation euphémique comme "obstacles techniques " pour dénommer des barbelés n'est pas assumée du fait qu'elle est tenue pour " difficilement compréhensible»:

[14] La Slovaquie a annoncé hier son intention de mettre en place des «obstacles techniques» - euphémisme difficilement compréhensible et maladroit - pour mieux contrôler l'arrivée des migrants (Le Matin du 12/11/2015).

En outre, évalué à l'aune d'un point de vue référentiel, l'euphémisme est couramment réprouvé comme étant fallacieux et à la source d'une désinformation qui contrecarre les principes de base de la déontologie journalistique. Entre autres, la dé-

${ }^{21}$ Source de points de vue, l'énonciateur - au sens de Ducrot (1984) - se fait évaluateur dès qu'il porte un jugement (axiologique, esthétique ou autre) sur une réalité donnée. 
nomination euphémique "globalisation " peut se voir non ratifiée, étant appréhendée comme « un nom trompeur»:

[15] Il est temps que l'on se donne les moyens de mettre fin aux perversions nées de cette globalisation qui ne fait que cacher, sous un nom trompeur et séduisant, les dérives du libéralisme (Jean-Paul Carteron, "Courrier des lecteurs », Le Temps du 23/06/2000).

Ou un verbe au sémantisme peu marqué, tel que "neutraliser " pour "tuer ", est considéré comme du « double langage » nuisant à la vérité, ce qui motive sa non prise en charge :

[16] Pour tuer, on dit «neutraliser ». Le double langage est tellement ancré dans la presse que, quand la vérité apparaît par miracle, elle fait l'effet d'une bombe (chronique de Frédéric Koller, Le Temps du 03/04/2012).

Sur un autre plan, envisageant un euphémisme selon un point de vue sociodiscursif en liaison avec la politesse, le journaliste peut s'en distancier, estimant qu'il porte paradoxalement atteinte à l'image des personnes qu'il prétend réhabiliter. Soit cet extrait du Point:

[17] On s'étonne du succès de la nouvelle expression à la mode, LGBT, pour dénommer les lesbiennes, les gays, les bi- et les transsexuels. Loin d'en améliorer la désignation, elle les transforme en une sorte de matricules anonymes du plus mauvais effet (Le Point du 15/05/2016).

Résultant de stratégies d'évitement et de réparation ${ }^{22}$ qui se traduisent par la substitution d'un sigle neutre à la dénomination discriminatoire de diverses minorités sexuelles, la formulation euphémique " $\mathrm{LGBT}^{23}$ " répond chez ses initiateurs à une volonté de revaloriser une communauté marginalisée et de favoriser une communication consensuelle à son sujet. Mais cet article du Point fait apparaître une polyphonie divergente dans ce dispositif énonciatif ${ }^{24}$, en mettant en relief un hiatus entre le point de vue d'intégration sociale visé et le point de vue dépersonnalisant auquel aboutit la siglaison, lequel est donné comme prépondérant.

Par ailleurs, la distanciation polyphonique à l'encontre d'un euphémisme, surtout dans le domaine de la guerre, s'appuie fréquemment sur des points de vue

22 Ces stratégies correspondent respectivement à la politesse négative et positive d'après KerbratOrecchioni (2005). Pour les rapports entre euphémisme et politesse négative, voir aussi Allan \& Burridge (2006).

${ }^{23}$ Bien qu'elle ne repose pas sur un substitut adoucissant, cette formulation est en effet euphémique par le simple fait qu'elle occulte des lexèmes désignant des groupes discriminés.

${ }^{24}$ Ce dispositif est lui-même ambigu, en ce qu'il émane d'un « on » qui peut inclure un nombre indéfini d'énonciateurs, dont le journaliste. 
éthiques, glissant vers la morale. C'est ainsi qu'un éditorialiste du Quotidien jurassien de Delémont refuse de prendre en charge la vision rassurante offerte par ces euphémismes militaires, la mettant sur le compte d'un positionnement hypocrite :

[18] Les mots font la guerre et la langue en crève. [...] Pour masquer l'horreur des combats, les bombardements ont des «frappes " aussi anonymes que les avions "furtifs" qui en ont la charge. [...] L'hypocrisie est ici non plus l'hommage du vice à la vertu, mais le refus de termes qui rappellent les horreurs du siècle (éditorial d'Albert Zbinden, Le Quotidien jurassien du 27/04/2005).

Ou encore la vision technicienne que ces euphémismes induisent est rejetée comme manifestant un positionnement cynique, source d'une manipulation mentale par laquelle les pouvoirs en place confortent leur emprise :

[19] Cautionnées par les gouvernements de la coalition auprès des opinions publiques, les frappes soi-disant chirurgicales ${ }^{25}$ sont banalisées avec un cynisme déterminé (Patrizia Farina, "Courrier des lecteurs ", Le Temps du 29/05/2011).

À travers la variété de leurs motivations, les clivages polyphoniques que nous avons mis en lumière révèlent des désaccords profonds sur le traitement médiatique des référents à risque, tout en posant la question de l'acceptabilité même des euphémismes dans la presse écrite.

\section{Polyphonie divergente et stratégies argumentatives à l'encontre des euphémismes}

Par-delà ses motivations énonciatives, la polyphonie divergente mettant en cause les euphémismes s'intègre dans des stratégies argumentatives qui visent à démonter leur mécanisme et à dévoiler leur artifice, tout en restaurant les expressions littérales qu'ils masquent. Le but est de réévaluer à la baisse les points de vue mélioratifs conférés à l'énonciation euphémique et d'assurer son échec dans la communication médiatique. Celui-ci commence dès que les formulations euphémiques ne sont plus reçues dans leur usage référentiel, mais qu'elles sont retraitées comme des mentions inadéquates en face de réalités problématiques. Sur le plan polyphonique, ces procédures argumentatives se traduisent par la confrontation d'un point de vue agent surplombant, autrement dit le point de vue critique, et d'un point de vue patient minoré, à savoir celui qui est associé à l'euphémisme. Une telle confrontation provoque la neutralisation de ce dernier moyennant un travail de sape qui revêt deux formes dans la presse écrite.

${ }^{25}$ Inséré dans la formulation euphémique " frappes chirurgicales », le marqueur « soi-disant » en implique ici la fausseté. 


\subsection{Stratégies de démasquage}

La première forme, à dominante rationnelle, est celle du démasquage ${ }^{26}$ qui consiste à identifier un euphémisme et à le disqualifier par un commentaire selon trois procédés. Le plus simple d'entre eux est le recours au métalangage rhétorique, comme dans un reportage du Matin consacré à la situation catastrophique au Zimbabwe :

[20] Dans le centre de Harare, la capitale, la police rafle les mendiants pour les emmener dans les quartiers à haute densité de population. C'est l'euphémisme développé par la langue de bois de l'administration pour désigner ce qu'on appelle "township " en Afrique du Sud (Le Matin du 14/12/2013).

Dans ce passage, les vocables "euphémisme", discrédité auprès de l'opinion ${ }^{27}$, et "langue de bois ", connoté négativement ${ }^{28}$, suffisent pour indiquer la réticence du journaliste et pour engager le lecteur à ne pas accorder du crédit à la périphrase édulcorante "quartiers à haute densité de population» utilisée par l'administration zimbabwéenne. L'emploi de cette terminologie métalinguistique fonctionne comme un dispositif d'alerte médiatique, en ce qu'un euphémisme nommé en tant que tel perd son efficacité pragmatique auprès du public. Au niveau polyphonique, on assiste à la confrontation entre un point de vue dissimulateur dénoncé (celui de l' "euphémisme ") et un point de vue factuel (la réalité des townships) mis en saillance par le journaliste, ce dernier point de vue étant présenté comme devant prédominer.

Une autre stratégie de démasquage consiste à utiliser des reformulations métadiscursives afin de rétablir le terme approprié, occulté par l'euphémisme. Le retour à un "parler juste" (selon l'expression de Koren, 1996 : 171) se fait à l'aide de formules rectificatrices, notamment pour les euphémismes portant sur le problème des réfugiés :

[21] Selon l'Office Fédéral des Migrations, chaque vol "spécial» - c'est-à-dire d'expulsion - affrété par la Suisse seule lui coûte entre 60000 et 100000 francs (24 heures du 05/12/2016).

On remarque aussi des reformulations par éclairage diachronique qui restituent le terme voilé, en particulier pour certains euphémismes dénommant des catégories sociales dévalorisées :

\footnotetext{
${ }^{26}$ À propos de cette stratégie liée à l'euphémisme, voir Bonhomme (2012).

${ }^{27}$ Un tel discrédit s'explique par les stéréotypes négatifs (dissimulation, tromperie, etc.) ordinairement associés au terme "euphémisme ", ce qui n'est pas le cas pour d'autres figures comme la métaphore ou la litote.

${ }^{28}$ Pour les affinités entre euphémisme et langue de bois, voir López Díaz (2014).
} 
[22] Monsieur le curé de Tournon partage le modeste presbytère avec un confrère. Ils disposent d'une "aide-aux-prêtres " [autrefois on disait plus précisément : bonne de curé] (Le Figaro du 24/12/2013).

La polyphonie divergente qui recouvre ces énoncés repose sur une stratégie d'explication: le je-dis correctif des journalistes s'exerce en vertu d'une éthique de l'adaptation du langage au monde. Cette éthique les conduit à déconstruire les effets modérateurs des tournures euphémiques et à rétablir les dénominations convenant aux situations exposées, en-deçà du dire jugé partial ou approximatif des énonciateurs euphémisants. Ces reformulations métadiscursives sont davantage tranchées lorsqu'il existe une grande distance entre l'euphémisme et les faits relatés, comme l'illustre l'occurrence suivante rapportant les déclarations d'un militant tiers-mondiste allemand à propos du drame des migrants en Méditerranée :

[23] «Il y en a qui parlent d'accidents en Méditerranée. Ce ne sont absolument pas des accidents, mais des crimes [...] qui se produisent soi-disant en notre nom ", a expliqué Philipp Ruch lors d'une conférence de presse (Le Matin du 20/06/2015).

Cette occurrence comporte une forte dislocation syntaxique, avec un présentatif initial ("Il y en a qui »), suivi de l'euphémisme dénoncé, et un second présentatif négatif ( Ce ne sont pas»), lui-même étant renforcé par le modificateur "absolument ». Ce dernier met en relief la reformulation de démasquage consécutive. Dans ce contexte, la critique de l'euphémisme se développe à travers l'antithèse entre le point de vue relativiste du on-dit euphémique ("accidents») et le point de vue délictuel («crimes»), rectificateur et à ce titre surplombant, du militant cité. De plus, suggérant une intention coupable, cette réalité criminelle est accentuée par sa formulation en position contre-argumentative après " mais ».

La mise en cause polyphonique des euphémismes s'effectue encore dans la presse écrite par le biais de stratégies présuppositionnelles plus indirectes ${ }^{29}$, comme le montrent ces deux exemples :

[24] Les notions telles que optimisation et assouplissement du marché du travail cachent souvent des idées comme celle d'un remplacement des engagements régis par le droit public par des contrats de droit privé (Le Temps du 07/03/2013).

[25] Le Kremlin prétend mener une guerre propre (titre de Paris Match du 21/10/2002).

Les euphémismes qui font l'objet de ces énoncés cristallisent dans leur posé un point de vue invariablement rehaussant sur la réalité qu'ils désignent, qu'elle soit économique ou militaire : il y a une amélioration du marché du travail; il y a une

\footnotetext{
${ }^{29}$ On trouvera dans Biglari \& Bonhomme (2018) une présentation détaillée de ces stratégies.
} 
guerre menée dans les règles en Tchétchénie. Toutefois, les verbes présuppositionnels qui les régissent dans le discours journalistique laissent entendre un point de vue réfutatif à travers lequel la vision positive qu'ils véhiculent est soit réévaluée comme suspecte, cacher ayant comme présupposé dissimuler des faits, soit niée, prétendre présupposant ne pas dire la vérité.

\subsection{Stratégies de stigmatisation}

Au degré fort, l'euphémisme se trouve non seulement démasqué, mais stigmatisé à l'issue d'un traitement polémique qui en fait ressortir la dimension manipulatoire. La stigmatisation de l'euphémisme adopte parfois la stratégie de l'ironie, à l'image de cet extrait dans lequel un journaliste du Temps fait semblant d'apprécier l'euphémisme "nettoyer " employé pour référer aux velléités génocidaires de certains responsables politiques russes à l'encontre des Tchétchènes :

[26] Autrefois, les autorités russes parlaient vrai quand elles disaient que tous les Tchétchènes devaient être éliminés du Caucase. Aujourd'hui les politiciens le disent d'une plus jolie manière: « Nous devons les nettoyer " (Le Temps du 02/10/2012).

Dans ce passage, le jeu polyphonique s'articule sur des contrastes de nature axiologique (valorisation des " autorités russes » vs dévalorisation des "politiciens ") et surtout d'ordre véridictoire, avec l'opposition entre la franchise passée du discours russe ("éliminer») et son édulcoration euphémique présente ("nettoyer »). Par l'antiphrase " jolie manière », le journaliste feint d'adhérer à ce point de vue mélioratif, mais le contexte de l'article (massacres en Tchétchénie) invite le lecteur à restituer son point de vue réprobateur implicite. À travers l'ambivalence de l'ironie, la fracture polyphonique s'incruste ainsi au cœur même de la parole journalistique. On relève une stratégie ironique voisine dans un article du Canard enchaîné qui dénonce le discours euphémique lénifiant du monde de l'entreprise :

[27] Source inépuisable d'euphémismes sirupeux, le monde de l'entreprise se gargarise du discours « bizounours » où tout le monde est bienveillant. C'est le constat du magazine Stratégies (5/10) remarquant que "talents» a remplacé "collaborateurs». Autres perles du glossaire : «appliquer le process » signifie « faire ce qui est demandé par un supérieur hiérarchique " et « licenciement» est remplacé par " nouvelle aventure ».Veinard de licencié qui va pouvoir profiter de son process! (Le Canard enchaîné du 11/10/2017).

En plus de l'antiphrase ("veinard de licencié»), cet article recourt à l'ironie par mention - au sens de Sperber et Wilson (1978) - à la dernière ligne : «qui va pouvoir profiter de son process". La polyphonie divergente se déploie alors entre le on-dit euphémique du jargon des entrepreneurs à la mode (" appliquer le process ") et le je-dis ironique du journaliste qui reprend ce jargon pour s'en distancier. 
La stigmatisation de l'euphémisme est beaucoup plus violente lorsqu'elle exploite les procédés pathémiques du pamphlet dégagés par Angenot (1982), en particulier quand elle s'en prend à la langue de bois appliquée aux conflits sociaux et armés. En atteste ce passage d'un éditorial de Philippe Castella dans La Liberté, dont l'objectif est de saper l'euphémisme "migrant", actuellement en pleine expansion pour désigner les réfugiés de la guerre et de la pauvreté :

[28] Le débat en Europe sur la question des migrants est en train de prendre une tournure folle. On a commencé par construire ce concept fourre-tout, ce monstre juridique, qui ne veut rien dire et efface la différence entre immigration économique et politique - la fameuse « misère du monde » avec les survivants de l'oppression, de la terreur, des massacres, à l'endroit desquels nous avons un devoir d'hospitalité (La Liberté du 27/08/2015).

Dans cet extrait, l'euphémisme " migrant " se voit mis à mal par l'exhibition de sa nature hétéroclite (" ce concept fourre-tout»), par sa recatégorisation au moyen d'une métaphore tératologique ("monstre»), par des jugements véhéments ("tournure folle ») et par des termes exacerbés ("terreur ", " massacres »). Totalement dysphorique, ce discours affectif-conflictuel ${ }^{30}$ met en scène une tension polyphonique fondée sur une incompatibilité entre le point de vue indigné de l'éditorialiste et le point de vue désensibilisé transmis par l'hyperonyme "migrant ${ }^{31}$ ".

\section{Conclusion}

Si l'on évalue les résultats de notre étude réalisée à partir d'un corpus de presse représentatif, on mesure la complexité de la polyphonie divergente liée à la mise en cause des euphémismes dans le discours journalistique. Cette complexité concerne ses relations fluctuantes avec le dialogisme interdiscursif et interlocutif, la pluralité de ses modalités discursives et de ses marqueurs énonciatifs, ainsi que la diversité de ses motivations et des stratégies argumentatives qu'elle mobilise. Cependant, il est possible de dégager quelques constantes qui confèrent une homogénéité d'ensemble au fonctionnement de la polyphonie divergente dans les euphémismes de presse. D'un côté, on a relevé la forte conflictualité des points de vue énonciatifs qu'elle confronte, essentiellement sur la tension et l'axiologie du discours. D'un autre côté, on a constaté l'orientation immuable des procédures de cette polyphonie divergente, puisqu'elles consistent toujours à déstabiliser le point de vue édulcorant des euphémismes par un point de vue rectificatif, plus conforme à la pertinence de

\footnotetext{
${ }^{30}$ Ce type de discours polémique a été décrit par Windisch (1987).

${ }^{31}$ Pour la caractérisation hyperonymique de ce terme, on peut se reporter à la définition qu'en donne Le Temps (31/08/2015) : "Migrant: Terme générique qui désigne celui qui quitte son pays, sans spécifier pour quelle raison ".
} 
l'information journalistique. De même, on a observé la permanence de sa visée perlocutoire, étant donné qu'il s'agit dans tous les cas de contribuer à l'échec des euphémismes pris pour cible, même s'il n'est jamais garanti d'avance. Enfin, on a pu percevoir une certaine uniformité thématique dans les euphémismes qu'elle met en question, ceux-ci portant principalement sur des problèmes militaires, sociaux et économiques. Au bout du compte, la polyphonie divergente à l'œuvre dans la presse écrite témoigne d'une réaction salutaire de la part de celle-ci contre le préformatage déceptif de la pensée euphémique, source d'une désinformation qui présente au public des jugements faussement rassurants comme allant de soi. Ce faisant, elle dément l'idée d'un positionnement énonciatif majoritairement conformiste qu'on voit trop souvent dans l'écriture journalistique.

\section{RÉFÉRENCES BIBLIOGRAPHIQUES}

AGNÈs, Yves (2002): Manuel de journalisme. Paris, La Découverte.

Allan, Keith \& Kate BuRRIDGE (2006): Forbidden Words. Cambridge, Cambridge University Press.

Amossy, Ruth (2005): "De l'apport d'une distinction: dialogisme vs polyphonie dans l'analyse argumentative », in J. Bres, P.-P. Haillet, S. Mellet, H. Nølke \& L. Rosier (éd.), Dialogisme et polyphonie. Approches linguistiques. Bruxelles, De Boeck/Duculot, 63-73.

ANGENOT, Marc (1982) : La Parole pamphlétaire. Paris, Payot.

AUTHIER-REVUZ, Jacqueline (1982) : "Hétérogénéité montrée et hétérogénéité constitutive : éléments pour une approche de l'autre dans le discours ». DRLAV, 26, 91-151.

AUTHIER-REVUZ, Jacqueline (1996) : "Remarques sur la catégorie de l'“îlot citationnel” ". Cahiers du français contemporain, 3, 91-116.

BIGLARI, Amir \& Marc BONHOMme (2018) : "Introduction", in A. Biglari \& M. Bonhomme (éd.), La Présupposition entre théorisation et mise en discours. Paris, Classiques Garnier, 7-31.

Bonhomme, Marc (2005) : Pragmatique des figures du discours. Paris, Honoré Champion.

BONHOMME, Marc (2012) : "La réception de l'euphémisme : entre réussite et échec interactif ", in M. Bonhomme, M. de La Torre \& A. Horak (éd.), Études pragmaticodiscursives sur l'euphémisme. Frankfurt am Main, Peter Lang, 73-88.

BONHOMME, Marc, Mariela DE LA TORRE \& André HORAK [éd.] (2012) : Études pragmatico-discursives sur l'euphémisme. Frankfurt am Main, Peter Lang.

BONHOMME Marc (sous presse) : "Ilots textuels euphémiques et polyphonie divergente". Communication présentée à la journée d'étude Quand les euphémismes s'affichent 
dans le discours (Saint-Jacques-de-Compostelle, 7 février 2020), à paraître dans Studia Neophilologica.

BRES, Jacques (2005) : «Savoir de quoi on parle : dialogue, dialogal, dialogique, dialogisme, polyphonie », in J. Bres, P.-P. Haillet, S. Mellet, H. Nølke \& L. Rosier (éd.), Dialogisme et polyphonie. Approches linguistiques. Bruxelles, De Boeck/Duculot, 47-61.

CASAS GÓMEZ, Miguel (2005) : «Precisiones conceptuales en el ámbito de la interdicción lingüistica ", in L. Santos Río (éd.), Palabras, norma, discurso. Salamanca, Ediciones Universidad de Salamanca, 271-290.

COURLESCU, Steluța (2014) : "Dialogisme et polyphonie dans le discours argumentatif». Analele Universitatii Crestine Dimitrie Cantemir, 2, 41-53.

COURTHÉOUX, Jean-Pierre (2005) : La Socio-euphémie. Paris, L'Harmattan.

DÉTRIE, Catherine, Paul SIBLOT \& Bertrand Verine (2001): Termes et concepts pour l'analyse du discours. Une approche praxématique. Paris, Honoré Champion.

DRUETTA, Ruggero \& Paola PAISSA [éd.] (2009) : Synergies Italie, numéro spécial : euphémismes et stratégies d'atténuation du dire. URL: https://gerflint.fr/Base/Italiespecial/italiespecial.html.

DuCrot, Oswald (1984) : Le Dire et le dit. Paris, Minuit.

JAMET, Denis \& Manuel JOBERT [éd.] (2010) : Empreintes de l'euphémisme. Tours et détours. Paris, L'Harmattan.

JAUBERT, Anna (2008) : " Dire et plus ou moins dire. Analyse pragmatique de l'euphémisme et de la litote ". Langue française, 160, 105-116.

Kerbrat-Orecchioni, Catherine (2005) : Le Discours en interaction. Paris, Armand Colin.

KOREN, Roselyne (1996) : Les Enjeux éthiques de l'écriture de presse et la mise en mots du terrorisme. Paris, L'Harmattan.

KRIEG-Planque, Alice (2003) : "Purification ethnique ». Une formule et son histoire. Paris, CNRS Éditions.

KRIEG-PlanQUE, Alice (2004): "Souligner l'euphémisme: opération savante ou acte d'engagement? Analyse du "jugement d'euphémisation" dans le discours politique ». Semen, 17, 59-79.

KRIEG-PlANQUE, Alice (2009) : La Notion de "formule» en analyse du discours. Besançon, Presses Universitaires de Franche-Comté.

LÓPEZ DÍAZ, Montserrat (2014) : "L'euphémisme, la langue de bois et le politiquement correct ». L'Information grammaticale, 143, 47-55.

LÓPEZ DÍAZ, Montserrat \& Jean-François SABLAYROLLES [éd.] (2016) : Les néologismes euphémiques. La Linguistique, 52.

MOIRAND, Sophie (2002) : «Dialogisme », in P. Charaudeau \& D. Maingueneau (éd.), Dictionnaire d'analyse du discours. Paris, Le Seuil, 175-178. 
PERRIN, Laurent (2004) : «Polyphonie et autres formes d'hétérogénéité énonciative : Bakhtine, Bally, Ducrot, etc. ». Pratiques, 123-124, 7-26.

PougeOISE, Michel (2001) : Dictionnaire de rhétorique. Paris, Armand Colin.

RABATEL, Alain (2006) : "La dialogisation au cœur du couple polyphonie/dialogisme chez Bakhtine ». Revue romane, 41/1, 55-80.

SPERbER, Dan et Deirdre WILSON (1978) : «Les ironies comme mentions ». Poétique, 36, 399-412.

VION, Robert (1992) : La Communication verbale. Paris, Hachette.

WINDISCH, Uli (1987) : Le K.-O. verbal. Lausanne, L'Âge d'Homme. 